54）宮原是中, 佐渡友㢁夫：化学工学, 35 (12), 1347 (1971)

55）宮原是中, 佐渡友秀夫：同上, 36 (7), 792 (1972)

56) Morisue, T., K. Noda and K. Ishida : J. Chem. Eng. Japan, 5 (3), 219 (1972)

57) Nagata, I., T. Ota and T. Takahashi : ibid., 5 (3), 227 (1972)

58) Nagata, I. and T. Ota: ibid., 5 (3), 232 (1972)

59) Nagata, I.: ibid., 6 (1), 18 (1973)

60) Nagata, I. and T. Ohta: J. Chem. Eng. Data, 16 (2), 164 (1971)

61）長浜邦雄，須田精二郎，白田利膦，平田光穂：石油触, 14 (4), 252 (1971)

62) Nishi, Y.: J. Chem. Eng. Japan, 5 (4), 334 (1972)

63) 西泰英: 化学工学, 35 (11), 1257 (1971)

64) 西泰英: 同上, 35 (4), 401 (1972)

65) Ochi, K. and K. Kojima: J. Chem. Eng. Japan, 4 (2), 122 (1971)

66) Ochi, K., M. Kato and K. Kojima: ibid., 4 (4), 301 (1971)

67）越智健二, 小島和夫：化学工学, 35 (5), 583 (1971)

68) Packer, L. G., S. R.M. Ellis and L.D.J. Soares: Chem. Eng. Sci., 28 (2), 597 (1973)

69) Palmer, D.A. and B.D. Smith: Ind. Eng. Chem., Process Design Develop. 11 (1), 114 (1972)

70) Palmer, D. A., W.I.C. Yuan and B.D. Smith: Ind. Eng. Chem., Fundamentals, 11 (3), 397 (1972)

71) Palmer, D.A. and B.D. Smith: J.Chem. Eng. Data, 17 (1), 71 (1972)

72) Perillox, C. J. and H. A. Deans: Ind. Eng. Chem., Fundamentals, 11 (1), 138 (1972)

73) Peter, S. and H. Wenzel: Chem. Ing. Techn., 43 (10), 623 (1971)

74) Raal, J.D., R.K. Code and D.A. Best: J. Chem. Eng. Data, 17 (2), 211 (1972)

75) Ramalho, R.S. and J.F. Drolet: ibid., 16 (1), 12 (1971)

76) Rong, M. and G.A. Ratcliff : Can. J. Chem. Eng., 49 (6), 825 (1971)

77) Ruether, J.A. and B.C.-Y. Lu: ibid., 50 (2), 266 (1972)

78) Sadler, L. Y, III., D.W. Luff and M.D. McKinley: $J$. Chem. Eng. Data, 16 (4), 446 (1971)

79) Sagnes, M. and V. Sanchez: ibid., 16 (3), 351 (1971)
80) Samuels, M.R.: Ind. Eng. Chem., Fundamentals, 11 (3), 422 (1972)

81) Samuels, M.R., D. L. Ulrichson and F.D. Stevenson: $A$. I. Ch. E. Journal, 18 (5), 1004 (1972)

82) Scheller, W.A., J. L. Petricek and G.C. Young: Ind. Eng. Chem., Fundamentals, 11 (1), 53 (1972)

83) Schreiber, L. B. and C.A. Eckert: Ind. Eng. Chem., Process Design Develop., 10 (4), 572 (1971)

84) Sokolov, N.M., N. N. Sevryugova and A. N.M. Zhavoronkov: Inst. Chem. Engrs. Symp. Ser. No.32, p.3: 110, Inst. Chem. Engrs., London (1969)

85) Strubl, K., V. Svoboda and R. Holub : Collection Czechoslov. Chem. Commun., 37 (11), 3522 (1972)

86) Strubl, K., P. Voňka, V. Svoboda and R. Holub: ibid., 38 (2), 468 (1973)

87) Suska, J., J.P. Novák, J. Matouš and J. Pick : ibid., 37 (8), 2664 (1972)

88) Tai, B. T., R.S. Ramalho and S. Kaliaguine: Can. J. Chem. Eng., 50 (6), 771 (1972)

89) Tao, L.C.: Inst. Chem. Engrs. Symp. Ser. No. 32, p. 3: 35, Inst. Chem. Engrs., London (1969)

90) Tassios, D.: A. I. Ch. E. Journal, 17 (6), 1367 (1971)

91) Tassios, D.P.: Ind. Eng. Chem. Process Design Develop., 11 (1), 43 (1972)

92）栃木勝己, 筩藤佳昭, 小島和夫：化学工学, 35 (6), 634 (1971)

93) Tsonopoulos, C. and J.M. Prausnitz: Ind. Eng. Chem., Fundamentals, 10 (4), 593 (1971)

94）内山 久：蒸留技行，1（1３）(1971)， 2 (1) (1972)

95) Ulrichson, D. L. and F.D. Stevenson: Ind. Eng. Chem., Fundamentals, 11 (3), 287 (1972)

96) Van Ness, H.C., S. M. Byer and R.E. Gibbs : A. I. Ch. E. Journal, 19 (2), 238 (1973)

97) Vera, J.H. and J.M. Prausnitz: J. Chem. Eng. Data, 16 (2), 149 (1971)

98) Wiehe, I. A., S. Dorai, C. G. Rader and A. Chandrasekhar: Chem. Eng. Sci., 26 (6), 901 (1971)

99) Wong, K.F. and C.A. Eckert: Ind. Eng. Chem., Fundamentals, 10 (1), 20 (1971)

100) Yuan, W.I.C., D. A. Palmer and B.D. Smith: ibid., 11(3), 387 (1972)

\title{
2. 高压気液平衡
}

\section{吉 村 尚 真**}

\section{1. 測定関係}

高圧気液平衡の测定に 関する研究の 歴史は 1869 年の Andrews $^{\mathrm{A}-17)}$ の炭酸ガスの $\mathrm{P}-\mathrm{V}-\mathrm{T}$ に関する研究とか, 1880 年の Cailletet ${ }^{\mathrm{C}-21)}$ の逆行凝縮に関する研究などを 初期のすのとみなせば約一世紀に亘るが，活発に行なわ れるようになったのは石油成分系の大量処理の必要性が 生じた 1930 年頃からであり，また我が国においては十

* 沿和 48 年 8 月 13 日受理

** Shoshin Yoshimura (正会員) 広島大学工学部
数年前からであり一般にそれ程古いるのではない。しか し近年工業用装置の高圧下の操業が進められるにつれて 研究は急速に進んでいる。

圧力範囲は工業的には 20〜50 気圧程度の場合が多く, 特に高い場合で 100〜150 気王であるが，測定データは Streett の $\mathrm{He}+\mathrm{CH}_{4}{ }^{\mathrm{s}-58)}$ 系の 10,000 気圧におよぶすの もある。一方, 温度範囲は大ざっぱに分類すると水添分 解関係 $\sim 500^{\circ} \mathrm{C}$, 石油精製関係 $\sim 200^{\circ} \mathrm{C}, \mathrm{NaOH}$ による

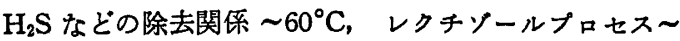


$-80^{\circ} \mathrm{C}, \mathrm{LNG}$ 関係 $-160^{\circ} \mathrm{C}$, 深冷分離, 空気分離関係 $-196^{\circ} \mathrm{C}$ ，極低温系などであるか，高温系 $\left(\sim 500^{\circ} \mathrm{C}\right)$ と 極低温系では測定データは非常に少く前者では $\mathrm{H}_{2}+$ 炭 化水素系の Grayson ${ }^{\mathrm{G}-13)}$, 後者では Streett $の \mathrm{Ne}+\mathrm{H}_{2} \mathrm{~s}-$ 55), $\mathrm{He}+\mathrm{H}_{2}{ }^{\mathrm{s}-34)}$ などである。

測定装置を測定型式により分類し（表 1 参照）それぞ れについていくつかの代表的文献をつぎに示す。循環型 ; Hurt ${ }^{\mathrm{H}-33)}$, 平田 $5^{\mathrm{H-16.28)}}$, Muirbrook $5^{\mathrm{M-22)}}$, Streett $5^{\text {s-32) }}$, Toyama $5^{T-17)}$, Thorogood $5^{T-3)}$, 頼実 $5^{\mathrm{x}-1921)}$, 露点沸点型; Ellington $5^{\mathrm{E}-12)}, \operatorname{Kay}^{\mathrm{K}-10)}$, 斉藤 $5^{\mathrm{A}-18,19)}$, 流通型; $\mathrm{Burch}^{\mathrm{B}-22)}$, 葛岡 $5^{\mathrm{K}-42)}$, Stutzman $5^{s-40)}$, Stetchel $5^{s-25)}$, 静置型; DePriester ${ }^{D-8)}$, 上西 $5^{\mathrm{K-36)}}$, Kohn $5^{5-25)}$, Saito $5^{5-44)}$, Zenner $5^{2}$ -3)。

装䡩の構成部設計に関する技術的一般参考書としては “高圧実験技術とその応用”N-11)，“Cryogenic Engineering"s-46), "Experimental Techniques in Low Temperature Physics"w-17) などがある。

装置および測定法に関する解説・レビューの類として は, Hǎla ら"-23) の “Vapor-Liquid Equilibrium” は, 1965 年ごろまでの各型の平衡セルを紹介しており, 平田 $5^{\text {日-25) }}$ の総説 “高圧気液平衡測定装置” はわが国最初の あの（1964）であり，これには，测定装置を原理的に前 記 4 大別し，6種を代表として取り上げ解説し，さらに 装置に詳しい文献もまとめてある。その他須田 ${ }^{\mathrm{S}-56)}$ の “高圧気液平衡”は実測上の問題を中心に各種の測定法が 検討されており，頼実ら ${ }^{\mathrm{Y}-22)}$ の“高王における気液平 衡の測定” は低温 $\left(\sim-196^{\circ} \mathrm{C}\right)$, 高圧 ( 370 気圧) に 主眼をおいている。レビューとしては物性定数 1 集 (1963) 10 集 $(1972)^{\mathrm{B}-36)}$ が各年ごとの諸事項要約付の データ文献一覧表をのせており, 小島 ${ }^{\mathrm{K}-41)}$, 片山ら ${ }^{\mathrm{K}-37)}$ はそれぞれ 1968，1968 年 1 月〜1971 年 3 月ごろまで の文献についてレビューしている。なお, 平田ら ${ }^{\text {B-34) }}$ “炭化水素の物性”（その 1〜8）はエンジニヤリング・デ ータとして貴重である。

実測データの収集としてはJ.C. Chu らの “VaporLiquid Equilibrium Data"(-30), "Distillation Equilibrium Data"C-29), Kogan 5の "Handbuch der DampfFlüssigkeits-Gleichgewichite” ${ }^{\mathrm{K}-40)}$, 化学工学協会編“物 性定数” 1 10 集 ${ }^{\mathrm{B}-36)}$ ，蒸留技術 No. 1 $7^{\mathrm{J}-4)}$ などがあ る。またデータの所在については平田・頼実編集の蒸留 工学ハンドブック ${ }^{\mathrm{H}-26)}$, 前述の Hăla らの 書物 ${ }^{\mathrm{B}-23)}$ な ぞがあり，低温関係に限定したものとして Ruheman ら R-39) $の$ "Bibliography of Vapor-Liquid Equilibrium of Low Boiling Mixtures and Notes on $K$-value Correlations"とか, 平田ら"-27) の炭化水素の物性 (その 1) 低温 に打ける気夜平衡関係などがある。石油誌上には平田ら
H-28,37 40) の炭化水素系に関する データが数多く発表さ れている。

今回調查した文献は下記のとおりであるが，紙面の都 合で全部は蒸留技術留話会編集の “蒸留技術” に近く発 表される予定である。

Chemical Abstract, 54, (17)(1960) 63, (2) (1965); 科学 技術文献速報，4（1）（1961）～8，(29）（1965）；A.I.Ch. E.J. 1, (1) (1955) 19, (2) (1973) [14, (1) 欠]; Appl. Sci. Res., 17, (1967) 27, (5) (1973); Bull. Japan Petrol. Inst., 13, (1) (1971) 14, (2) (1972); Brit. Chem. Eng.,12, (1) (1967) 17, (9) (1972); Process Technology International, 17, (10) (1972) 18, (3) (1973); Cryogenics, 5, (1) (1965) 13, (4) (1973); Chem. Eng., 75, (1)(1968) 80, (13) (1973); Chem. Eng. Progr., 63, (1) (1967) 69, (4) (1973) [64, (12) 欠] ; Chem. Eng. Sci., 22, (1) (1967) 28, (4) (1973); Can. J. Chem. Eng. 41, (1) (1967) 50, (6) (1972); Chem. Ing. Tech., 39, (1) (1967) 45, $(9+10)$ (1973); Hydrocarbon Processing, 47, (1) (1968) 52, (5) (1973); Ind. Eng. Chem., 59, (1) (1959) 62, (1970); Ind. Eng. Chem., Fundamentals, 6, (2)(1967) 12, (1)(1973); J.Chem. Eng. Data, 5, (1) (1960) 18, (2)(1973); J. Chem. Eng. Japan 1, (1)(1968) 0, (2) (1973); J. Chem. Thermodynamics, 1, (1) (1969) 5, (3) (1973); 化学工学, 34, (1) (1970) 37, (7) (1973); 石油誌 9, (1) 1966 15, (12)1972; Trans. Inst. Chem. Engrs. (London), 45，(1967) 49，(1971）および前 記の物性定数 $1 \sim 10$ 集 ${ }^{\mathrm{B}-36)}$, 蒸留技術ハンドブック Ruheman $5^{\mathrm{R}-39)}$, 平田ら日-27) の文献リストから高圧関係を抜 粋し付加した。またこれらの他に入手した文献を若干加えた。

衣 1 は上記文献の 1970 年以後のものを枝すいしたも のである。

\section{2. 理論 (推算) 関係}

高圧における気液平衡の推算は，初期においては理想 溶液を仮定して気液平衡比 $K を$ 相関図を用いて求める方

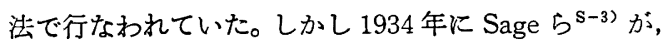
また 1938 年に $\mathrm{Kay}^{\mathrm{K}-6)}$ が，“ $K$ は温度と圧力が一定で も組成によって変る”ことを実験的に示して以来組成の 影響を考虑するために種々の方法が考えられ, Kellog チ +ート ${ }^{\text {K-39) }}$ とか, これに改良を加えたものとして Ed-

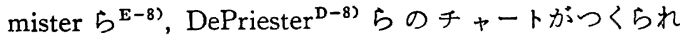
た。これらは組成パラメータとしてモル平均沸点を使用 したものであるが，1953 年組成パラメータとして, Had-

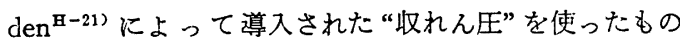
に, $\operatorname{NGPA}^{N-10)}$, Winn ${ }^{W-20)}$, Cajander $5^{L-22)}$, Grayson $5^{\text {H-22) }}$, Lenoir ${ }^{\mathrm{L}-21)}$, API $の^{\text {“Technical DataBook" }}$ "16), などがある。四解法の解説にはEdmister ${ }^{\mathrm{E}-9)}$, 平田 ${ }^{\mathrm{B}-26)}$ がある。

一方, 解析的な方法は 1940 年ごろから電算機が使用 され始めるとしたいに注目されるよらになり，これまた 数多くの方法があるが，幸いに熱力学的基礎からときお. 
表 1 Literatures of high pressure vapor-liquid equilibrium data

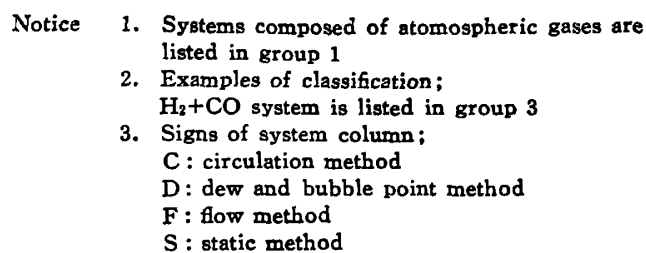

Group No.1 Atmospheric gases

\begin{tabular}{lccc} 
System & $\begin{array}{c}\text { Pressure range } \\
\text { [atm] }\end{array}$ & $\begin{array}{c}\text { Temperature } \\
\text { range }\left[{ }^{\circ} \mathrm{C}\right]\end{array}$ & Literature \\
\hline $\mathrm{Ar}+\mathrm{N}_{2}$ & $8.2 \sim 14.9$ & -161.2 & $\mathrm{M}-19$ \\
$\mathrm{~N}_{2}+\mathrm{He}$ & $\sim 4000$ & $-195.2 \sim-136.2$ & $\mathrm{~S}-37$ \\
$\mathrm{~N}_{2}+\mathrm{He}$ & $6.8 \sim 340.1$ & $37.8 \sim 176.7$ & $\mathrm{M}-5$ \\
$\mathrm{He}-\mathrm{Kr}$ & 120 & $-156 \sim-123.2$ & $\mathrm{~K}-20$ \\
$\mathrm{Ar}+\mathrm{N}_{2}+\mathrm{O}_{2}$ & $3.9 \sim 7.1$ & $-183.2 \sim-153.2$ & $\mathrm{E}-4$ \\
\hline
\end{tabular}

Group No.2 Hydrocarbon systems only 2-1) systems containing $\mathrm{CH}_{4}$

\begin{tabular}{lccl}
\hline $\mathrm{C}_{3} \mathrm{H}_{8}(\mathrm{C})$ & $1.7 \sim 63.9$ & $-142.8 \sim-59.4$ & $\mathrm{~W}-8$ \\
$n-\mathrm{C}_{5} \mathrm{H}_{12}$ & $68.1 \sim 136$ & 104.4 & $\mathrm{P}-13$ \\
$i-\mathrm{C}_{5} \mathrm{H}_{12}$ & $33.9 \sim 149$ & $71.1 \sim 137.8$ & $\mathrm{P}-13$ \\
$n e o-\mathrm{C}_{5} \mathrm{H}_{12}$ & $21 \sim 118.9$ & $71.6 \sim 104.4$ & $\mathrm{P}-13$ \\
$\mathrm{C}_{2} \mathrm{H}_{6}+\mathrm{C}_{3} \mathrm{H}_{8}+n-\mathrm{C}_{5} \mathrm{H}_{12}$ & $6.8 \sim 272$ & $65.6 \sim 121.1$ & $\mathrm{~V}-1$ \\
$+n-\mathrm{C}_{6} \mathrm{H}_{14}+n-\mathrm{C}_{10} \mathrm{H}_{22}$ & & &
\end{tabular}

2-2) systems containing $\mathrm{C}_{2} \mathrm{H}_{6}$

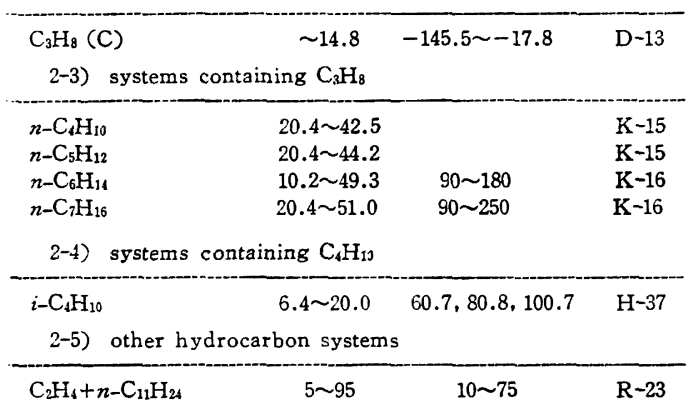

Group No. 3 Systems containing $\mathbf{H}_{2}$

\begin{tabular}{lccc}
\hline $\mathrm{N}_{2}$ & $16.5 \sim 188$ & $-185.0,-195.8$ & $\mathrm{Y}-21$ \\
$\mathrm{~N}_{2}$ & $34 \sim 370$ & 0 & $\mathrm{Y}-7$ \\
$\mathrm{CO}_{2}$ & $60 \sim 370$ & $60 \sim 370$ & $\mathrm{Y}-7$ \\
$\mathrm{CO}$ & $\sim 100$ & $\sim 100$ & $\mathrm{~J}-2$ \\
$\mathrm{CH} \mathrm{H}_{4}$ & $19.7 \sim 106.9$ & $-99.5 \sim-170$ & $\mathrm{~S}-1$ \\
$\mathrm{C}_{2} \mathrm{H}_{4}$ & $20 \sim 80$ & $-25 \sim-150$ & $\mathrm{~S}-1$ \\
$\mathrm{C}_{2} \mathrm{H}_{6}$ & $20 \sim 80$ & $-50 \sim-125$ & $\mathrm{~S}-1$ \\
$\mathrm{C}_{3} \mathrm{H}_{8}$ & 204.1 & $-180 \sim 75$ & $\mathrm{~T}-11$ \\
$\mathrm{CO}+\mathrm{C}_{3} \mathrm{H}_{8}$ & 204.1 & $-180 \sim 75$ & $\mathrm{~T}-11$ \\
$\mathrm{CO}+\mathrm{C}_{3} \mathrm{H}_{8}$ & $34 \sim 204.1$ & $-50 \sim 50$ & $\mathrm{~T}-11$ \\
$\mathrm{CH}_{4}+\mathrm{NH}_{3}$ & $\leqq 200$ & 25 & $\mathrm{~K}-29$ \\
$\mathrm{CH}_{4}+\mathrm{C}_{2} \mathrm{H}_{4}$ & $20 \sim 80$ & $-25 \sim-150$ & $\mathrm{~S}-1$ \\
$\mathrm{C}_{2} \mathrm{H}_{4}+\mathrm{C}_{2} \mathrm{H}_{5}$ & $20 \sim 80$ & $-50 \sim-125$ & $\mathrm{~S}-1$ \\
$\mathrm{~N}_{2}+\mathrm{CH}_{4}+\mathrm{NH}_{3}$ & $\leqq 200$ & 25 & $\mathrm{~K}-29$ \\
$\mathrm{CO}+\mathrm{CH}_{4}+\mathrm{CO}_{2}+\mathrm{C}_{3} \mathrm{H}_{8}(\mathrm{D}) \quad 8 \sim 42$ & 0 & $\mathrm{Y}-24$ \\
$\mathrm{CO}+\mathrm{CH}_{4}+\mathrm{CO}_{2}+n-\mathrm{C}_{4} \mathrm{H}_{10} \quad 9 \sim 45$ & 0 & $\mathrm{Y}-24$ \\
$(\mathrm{D})$ & & & \\
\hline
\end{tabular}

Group No.4 Systems containing $\mathrm{N}_{2}$

$\begin{array}{llll}\mathrm{CO}_{2} & -40 \sim 25 & \mathrm{~V}-1\end{array}$

\begin{tabular}{lccc}
$\mathrm{CO}_{2}$ & $34.4 \sim 116.7$ & 0 & $\mathrm{Y}-7$ \\
\hline & & & \\
\multicolumn{1}{c}{ Group No. 5 } & \multicolumn{4}{c}{ Systems containing CO } \\
\hline $\mathrm{O}_{2}$ & $\sim 130$ & $-50 \sim 10$ & $\mathrm{~F}-13$ \\
$\mathrm{O}_{2}$ & $7 \sim 523$ & -49.4 & $\mathrm{~F}-12$ \\
$\mathrm{O}_{2}$ & $10 \sim 130$ & $-50 \sim 10$ & $\mathrm{~F}-13$ \\
$\mathrm{He}$ & $\sim 139$ & $-20 \sim 20$ & $\mathrm{~B}-23$ \\
$\mathrm{CH}_{2} \mathrm{~F}_{2}$ & $1 \sim 44$ & $-51 \sim 10$ & $\mathrm{~A}-4$ \\
$\mathrm{CH}_{4}+\mathrm{C}_{10} \mathrm{H}_{22}$ & $7 \sim 119$ & $4 \sim 65$ & $\mathrm{Y}-8$ \\
\hline
\end{tabular}

\begin{tabular}{lrrr} 
& Group No.6 & \multicolumn{4}{c}{ Systems containing CO } \\
\hline $\mathrm{C}_{2} \mathrm{H}_{6}$ & $\sim$ critical & $-100 \sim 0$ & $\mathrm{~T}-10$ \\
$\mathrm{C}_{3} \mathrm{H}_{8}$ & -136.1 & $-125 \sim 50$ & $\mathrm{~T}-10$ \\
\hline
\end{tabular}

Group No. 7 Systems containing $\mathbf{H}_{2} \mathrm{~S}$

\section{Group No. 8 Miscellaneous}

\begin{tabular}{|c|c|c|c|}
\hline $\mathrm{H}_{2} \mathrm{O}+\mathrm{HCl}$ & 15 & & $K-5$ \\
\hline $\mathrm{H}_{2} \mathrm{O}+\mathrm{HCl}$ & $1.1 \sim 15.1$ & & $\mathrm{~K}-5$ \\
\hline $\mathrm{H}_{2} \mathrm{O}+\mathrm{CH}_{3} \mathrm{CN}+n-\mathrm{C}_{4} \mathrm{H}_{10}$ & 3.9 & & $P-2$ \\
\hline $\mathrm{H}_{2} \mathrm{O}+\mathrm{CH}_{3} \mathrm{CN}+\mathrm{C}_{4} \mathrm{H}_{8}$ & 4.6 & & $P-2$ \\
\hline $\mathrm{H}_{2} \mathrm{O}+\mathrm{CH}_{3} \mathrm{CN}+\mathrm{C}_{4} \mathrm{H}_{6}$ & 4.4 & & $P-2$ \\
\hline $\mathrm{CH}_{4} \mathrm{O}+\mathrm{C}_{3} \mathrm{H}_{6}$ & $2.2 \sim 9.5$ & 19.9 & $\mathrm{~N}-12$ \\
\hline $\mathrm{CH}_{4} \mathrm{O}+\mathrm{C}_{3} \mathrm{H}_{8}$ & $2.7 \sim 7.9$ & 19.9 & $\mathrm{~N}-12$ \\
\hline $\mathrm{CH}_{3} \mathrm{OH}+\mathrm{CH}_{3} \mathrm{COOCH}_{3}$ & $5.8 \sim 11.6$ & $312.7 \sim 140.2$ & $\mathrm{~N}-1$ \\
\hline $\mathrm{C}_{2} \mathrm{H}_{5} \mathrm{OH}+n-\mathrm{C}_{i} \mathrm{H}_{16}+\mathrm{C}_{6} \mathrm{H}_{6}$ & $0.3 \sim 1.2$ & 79 104 & $\mathrm{T}-12$ \\
\hline $\mathrm{C}_{2} \mathrm{H}_{5} \mathrm{OH}+\mathrm{C}_{3} \mathrm{H}_{6}$ & $1.7 \sim 9.4$ & 19.9 & $\mathrm{~N}-12$ \\
\hline $\mathrm{C}_{2} \mathrm{H}_{5} \mathrm{OH}+\mathrm{C}_{3} \mathrm{H}_{8}$ & $2.4 \sim 7.9$ & 19.9 & $\mathrm{~N}-12$ \\
\hline$n-\mathrm{C}_{3} \mathrm{H}_{8} \mathrm{O}+\mathrm{C}_{3} \mathrm{H}_{6}$ & $1.7 \sim 9.5$ & 19.9 & $\mathrm{~N}-12$ \\
\hline$n-\mathrm{C}_{3} \mathrm{H}_{8} \mathrm{O}+\mathrm{C}_{3} \mathrm{H}_{8}$ & $1.4 \sim 7.9$ & 19.9 & $\mathrm{~N}-12$ \\
\hline$n-\mathrm{C}_{4} \mathrm{H}_{10} \mathrm{O}+\mathrm{C}_{3} \mathrm{H}_{6}$ & $2.9 \sim 9.3$ & 19.9 & $\mathrm{~N}-12$ \\
\hline$n-\mathrm{C}_{4} \mathrm{H}_{10} \mathrm{O}+\mathrm{C}_{3} \mathrm{H}_{8}$ & $1.5 \sim 7.8$ & 19.9 & $\mathrm{~N}-12$ \\
\hline $\mathrm{CH}_{3} \mathrm{COCH}_{3}+\mathrm{C}_{3} \mathrm{H}_{6}$ & $1.7 \sim 8.6$ & 19.9 & $\mathrm{~N}-12$ \\
\hline $\mathrm{CH}_{3} \mathrm{COCH}_{3}+\mathrm{C}_{3} \mathrm{H}_{8}$ & $1.4 \sim 7.1$ & 19.9 & $\mathrm{~N}-12$ \\
\hline $\mathrm{CH}_{3} \mathrm{COC}_{2} \mathrm{H}_{5}+\mathrm{C}_{3} \mathrm{H}_{6}$ & $1.8 \sim 8.6$ & 19.9 & $\mathrm{~N}-12$ \\
\hline $\mathrm{CH}_{3} \mathrm{COC}_{2} \mathrm{H}_{5}+\mathrm{C}_{3} \mathrm{H}_{8}$ & $1.2 \sim 7.2$ & 19.9 & $\mathrm{~N}-12$ \\
\hline $\mathrm{He}+\mathrm{CH}_{4}$ & $68.0 \sim 258.5$ & $-179.2 \sim-81.2$ & $\mathrm{R}-22$ \\
\hline $\mathrm{He}+\mathrm{CH}_{4}$ & 68.0 & $-149.2 \sim-82.6$ & $\mathrm{D}-10$ \\
\hline $\mathrm{N}_{2} \mathrm{O}+\mathrm{N}_{2}$ & $4.0 \sim 80.9$ & $-60 \sim-20$ & $z-1$ \\
\hline $\mathrm{N}_{2} \mathrm{O}+\mathrm{CH}_{4}$ & $4.1 \sim 52.0$ & $-60 \sim-20$ & $z-1$ \\
\hline $\mathrm{N}_{2} \mathrm{O}+\mathrm{O}_{2}$ & $4.1 \sim 90.1$ & $-60 \sim 10$ & $z-1$ \\
\hline $\mathrm{Ar}+\mathrm{C}_{2} \mathrm{H}_{6}$ & $24.3 \sim 131.4$ & -157.7 & $E-5$ \\
\hline $\mathrm{AT}+n e 0-\mathrm{C}_{5} \mathrm{H}_{12}$ & $11 \sim 247.4$ & 50 & $\mathrm{R}-30$ \\
\hline $\mathrm{Ar}+\mathrm{CHClF}_{2}$ & $15 \sim 191.3$ & $0 \sim 75$ & $\mathrm{~N}-2$ \\
\hline $\mathrm{ClF}_{3}+\mathrm{C}_{2} \mathrm{Cl}_{2} \mathrm{~F}_{4}$ & $2.1 \sim 3.4$ & & $\mathrm{R}-18$ \\
\hline $\mathrm{ClF}_{3}+\mathrm{WF}_{6}$ & 3.42 & & $\mathrm{R}-21$ \\
\hline $\mathrm{ClF}_{3}+\mathrm{MoF}_{6}$ & 3.42 & & $\mathrm{C}-2$ \\
\hline $\mathrm{CH}_{1}+\mathrm{CHClF}_{2}$ & $16.2 \sim 104.0$ & $0 \sim 75$ & $\mathrm{~N}-2$ \\
\hline $\mathrm{CHF}_{3}+\mathrm{CClF}_{3}$ & $2.1 \sim 27.3$ & $-73.4 \sim 0$ & $S-28$ \\
\hline $\mathrm{C}_{2} \mathrm{Cl}_{2} \mathrm{~F}_{1}+\mathrm{MoF}_{6}$ & 3.42 & & $\mathrm{R}-17$ \\
\hline $\mathrm{UF}_{6}+\mathrm{WF}_{6}$ & 3.42 & & $\mathrm{R}-20$ \\
\hline $\mathrm{UF}_{6}+\mathrm{MoF}_{6}$ & 3.42 & & $R-19$ \\
\hline $\mathrm{Ar}+\mathrm{CH}_{6}+\mathrm{C}_{2} \mathrm{H}_{6}$ & $59.0 \sim 82.9$ & -157.7 & $E-5$ \\
\hline $\mathrm{He}+\mathrm{C}_{8} \mathrm{H}_{8}+\mathrm{C}_{6} \mathrm{H}_{16}+i-\mathrm{C}_{8} \mathrm{H}_{18}$ & $1.3 \sim 1.8$ & $26.7 \sim 82.2$ & $P-3$ \\
\hline
\end{tabular}




\section{表 2 理詅（推算）に関する る 文献}

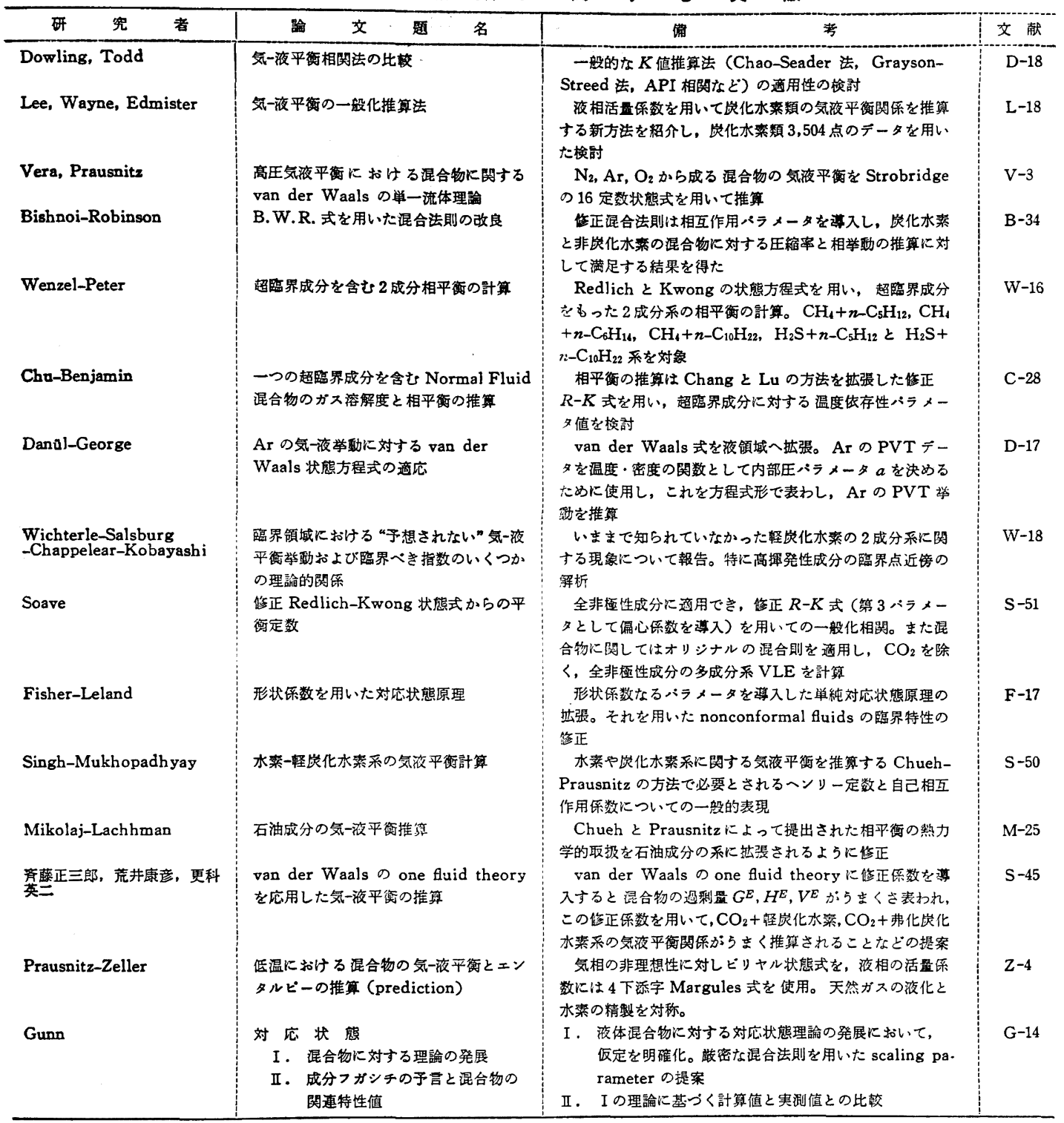

こした書物・解説・総説の類はかなり多く、これに属す るものとして Edmister ${ }^{\text {E-9)}}$, Prausnitz ${ }^{\text {P-14 16) }}$, Haselden $5^{8-52)}$, 平田 ${ }^{\mathrm{B}-26)}$, 頼実-吉村 ${ }^{\mathrm{Y}-122}$, 頼実-保岡 ${ }^{\mathrm{X}-15)}$ なと がある。

解析的な方法をらまく分類することは難しいが，ここ では 3 大別し Case I として状態式を用いる方法 (BWR 法 ${ }^{\mathrm{B}-31,32,37,38)}$ で代表される)，Case II として対応状態 原理に基つく方法 (Leland-Chappelear-Gamson ${ }^{\mathrm{L}-19)}$ の 方法で代表される）と，Case III として活量係数を用い る方法(Chao-Seader 法C-22) とか Prausnitz-Chueh ${ }^{\mathrm{C}-24 ~}$ 27)法などによって代表される）とする。Case I に関す
る推算法を報文として詳しく論じたものに 1964 年の平 田-中原 ${ }^{\mathrm{H}-24)}$ があり，推算の立場から BWR 式を扱った ものに頼実 ${ }^{\mathrm{T}-14)}$ がある。BWR 定数の決め方は四解法と しては原文 ${ }^{\mathrm{B}-31)}$ があり，最小二乗法によるものに Sage

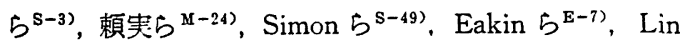
$ら^{\mathrm{L}-23)}$ がある。定数值表は, Cooper ${ }^{\mathrm{C}-31)}$, 頼実ら 13) によりまとめられ，定数の温度依存性に関しては Lee ${ }^{\mathrm{L}-16)}$, Price $5^{\mathrm{P}-17)}$, Eubanks ${ }^{\mathrm{E}-13)}$, Motard $5^{\mathrm{M}-211}$, Orye ${ }^{0-6)}$, Kaufmann ${ }^{\mathrm{K}-38)}$ などがある。定数の一般化は Edmister ${ }^{\mathrm{E}-10)}$ らが提案しておうり，系への適用は頼笑ら -7.18) が行っている。混合法則の修正を試みたものに 


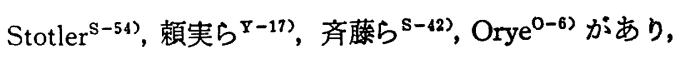
式の形の修正に関しては $\operatorname{Lin}^{\mathrm{L}-23)}$ がある。また我素十炭 化水素系への適用については平田-中原茥-35) によって論 しられ, 初期檤の与え方については中原-平田 ${ }^{\mathrm{N}-9)}$, 頼実一 如岡 ${ }^{\mathrm{Y}-16)}$ らの研究がある。また 4 成分系の成立範囲につ いては平田一中原 ${ }^{\mathrm{H}-36)}$ らの研究がある。BWR 式以外飞 Redlich-Kwong ${ }^{\mathrm{R}-36)}$ 式も式が簡単で系ごとの定数を用 いると精度が良いのでWilson $5^{\text {W-19), 頼実-脌岡ら }}$ によって使用されている。CaseII に関する研究は Lela$\mathrm{nd}^{\mathrm{L}-15,20)}$ により引続き行なわれているが，この方法を 改良し 発展させた研究成果を盛込んた解説に，頼実ら 又-12), 斉藤(-48) がある。前者は Hirschfelder-BuehlerMcGee-Sutton ${ }^{\text {H-29) }}$ の状態式を導入しており, 後者は 主として異種分子間相互作用パラメータに関するるの で，約 40 ページにわたる労作である。その他 Sather $ら^{\mathrm{F}-18)}$, Rowlinson $5^{\mathrm{R}-40)}$, Barbe $ら^{\text {B-29) }}$ の研究がある。 Case III の Chao-Seader 法に関するものに, $\mathrm{H}_{2}+$ 炭化水 素の $500^{\circ} \mathrm{C}$ 領域までの各温度に打液体フガシチ係 数計算式を誘導した Grayson ${ }^{(-13)} ら の$ 研究があり, Shelton $ら^{\mathrm{S}-47,48)}$ はフラッシュ計算, 露点・沸点計算へ の適用を詳しく論し， Robinson $5^{\mathrm{R}-38)}$ は $\mathrm{CH}_{4}+\mathrm{CO}_{2}+$ $\mathrm{C}_{4} \mathrm{H}_{10}, \mathrm{CH}_{4}+\mathrm{H}_{2} \mathrm{~S}+\mathrm{C}_{4} \mathrm{H}_{10}$ 系へこの方法の邀用を試みて いる。Prausnitz-Chueh らの方法は液相活量保数, 気相 フガシチ係数および夜相フガシチのそれぞれについて Chao-Seaderよりも䈯密な取り扱いをして扰り,“Computer Calculations for High-Pressure Vapor-Liquid Equi-
librium"P-15) 飞詳しく論しられており，また頼実一杵岡 又-15) によってる解説されている。その他 Case III に属 するものとして, Adler $5^{A-15)}$, Hoffman ${ }^{\mathrm{H-30}, 31)}$, Kobayashi $^{\mathrm{C}-3, \mathrm{v}-7)}$ らの研究がある。

高圧気液平衡理論のレビューには 1968 年以前の約 3 年間については小島曶-41), 1971 年以前の約 3 年間につい ては片山ら の論文については，すでに物性定数 1 10 集に収められ ているので, ここではその後の約 3 年間のものについて 主として推算関係のものを選び, 表 2 亿まとめた。調査 した文献とその範囲は以下に示すとおりである。

A. I. Ch. E. J., 17, (1)(1971) 19, (2)(1973); Appl. Sci. Res., 24, (1)(1971) 27, (5)(1973); Brit. Chem. Eng. (Pro. cess Technology International), 16, (1)(1971) 18, (3) (1973) ; Cryogenics, 11, (1) (1971) 13, (4) (1973); Chem. Eng. 78, (1) (1971) 80, (13) (1973); Chem. Eng. Progr., 67, (1) (1971) 69, (4) (1973); Chem. Eng. Sci., 26, (1)(1971) 28, (4)(1973); Can.J. Chem. Eng., 49, (1)(1971) 50, (6)(1972) ; Chem. Ing. Tech., 43, (1)(1971) 45, (9+10) (1973); Hydrocarbon Processing, 50, (1) (1971) 52, (5) (1973); Ind. Eng. Chem., Fundamentals, 9, (1) (1970) 12, (1) (1973); J. Chem. Eng. Data, 16, (1) (1971) 18, (2)(1973) ; J. Chem. Thermodynamics, 3, (1) (1971) 5, (3) (1973) ; Trans. Inst. Chem. Engrs. (London), 11, (1) (1971) 13, (2) (1973); J. Chcm. Eng. Japan, 4, (2) (1971) 6, (2) (1973); 化学工学, 34, (1) (1970) 37, (7) 1973; 石油誌, 13, (1) $(1970) \sim 15$, (12) (1972)

引用 文 献 (文献番号がとんでいるのは技すいしたためである)

A-4) Adams, R.A. and F.P.Stein: J. Chem. Eng. Data, 16 (2), 146-149 (1971)

A-15) Adler, S.B., H. Ozkardesh and W.C. Schreiner : Hydrocarbon Processing, 47 (4), 145 (1968)

A-16) American Petroleum Inst. "Technical Data Book, Petroleum Refining ${ }^{n}$, New York (1966)

A-17) Andrews, P.: Phil. Trans., 159, 575 (1869)

A-18）荒井康应, 上西玄一, 紊藤正三郎: J. Chem. Eng. Japan, 4, $113(1971)$

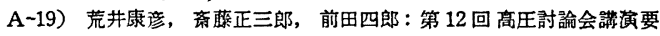
旨集, p. 115 (1970)

B-22) Burch, R. J.: J. Chem. Eng. Data, 2 (1), 19 (1964)

B-23) Burfield, D.W., H.P. Richardson and R. A. Guereca: A. I. Ch. E. J., 16 (1), 97 (1970)

B-29) Barbe, C., F. Dennery and B. Grison: "Symposium on Distillation", Brighton Eng! and, P. 108, Sept. (1969)

B-31) Benedict, M., G. B. Webb and L.C. Rubin: J. Chem. Phys., 8, 334 (1940)

B-32) Benedict, M., G. B. Webb, L.C. Rubin and L. Friend: Chem. Eng. Progr., 47, 571, 609 (1951)

B-34) Bishnoi, P. R. and D. B. Robinson: Hydrocarbon Processing, 51 (11), 152 (1972)

B-36）物性定数 1 集 10 隼，丸善 (1963 1972)

B-37) Benedict, M., G. B. Webb and L.C. Rubin: J. Chem. Phys., 10, 747 (1942)

B-38) Benedict, M., G. B. Webb and L.C. Rubin: Chem. Eng. Progr., 47, 449 (1951)
C-2) Carles, M. J. and J. Aubert: J. Chem. Phys., 67, 671 (1970)

C-3) Chang, H. L., L.J. Hurt and R. Kobayashi: A. I.Ch. E. $J, 12,1212$ (1966)

C-21) Cailletet, L.: Compt. Rend., 90, 210 (1880)

C-22) Chao, K.C. and J.D. Seader: A. I. Ch. E. J., 7, 598 (1961)

C-24) Cheuh, P.L. and J.M. Prausnitz: Ind. Eng. Chem., Fundamentals, 6, 492 (1967)

C-25) Cheuh, P.L. and J.M. Prausnitz: A. I. Ch. E. J., 13, 1099, 1107 (1967)

C-26) Cheuh, P.L. and J.M. Prausnitz: Hydrocarbon Processing, 47, 147 (1968)

C-27) Cheuh, P. L. and J. M. Prausnitz: Ind. Eng. Chem., 60 (3), 34 (1968)

C-28) Chu, H. and B.C.-Y. Lu: Can. J. Chem. Eng., 49 (Feb), 134 (1971)

C-29) Chu, J.C., et al.: "Distillation Equilibrium Data", Reinhold, New York (1950)

C-30) Chu, J.C., et al.: "Vapor-Liquid Equilibrium Data", J.W. Edwards, Michigan (1956)

C-31) Cooper, H.W. and J.C. Goldfrank: Hydrocarbon Processing, 46 (12), 141 (1967)

D-8) DePriester, C. L. : Chem. Eng. Prog. Symp. Ser, 49 (7), 1 (1953)

D-10) DeVaney, W.E., H. L. Rhodes and P.C. Tully: J. Chem. Eng. Data, 16 (2), 158 (1971) 
D-13) Djordjerich, L. and R.A. Budenholzer: J. Chem. Eng. Data, 15 (1), 10 (1970)

D-17) Danul, A. and G. Thodos : Can. J. Chem, Eng., 50 , June, 372 (1972)

D-18) Dowling, G.R. and W.G. Todd : Chem. Eng., March 19, 115 (1973)

E-4) Elshayal, I. M. and B.C.Y. Lu : J. Chem. Eng. Data, 16 (1), 31 (1971)

E-5) Elshayal, I. M. and B.C.-Y. Lu: Cry., 11 (4), 285(1971)

E-7) Eakin, B.H. and R.Y. Ellington: "Thermodynamic and Transport Properties of Gases, Liquid and Solid", ASME (New York, McGraw-Hill), 195 (1959)

E-8) Edmister, W.C. and C. L. Ruby: Chem. Eng. Progr., 51, 95-F (1955)

E-9) Edmister, W.C.: "Applied Hydrocarbon Thermodynamics", Gulf Pub. Co., Houston Texas (1961)

E-10) Edmister, W.C., J. Vairogs and A.J. Klekers : A. I. Ch. E. J, 14, 497 (1968)

E-12) Ellington, R. T., B.E. Eakin, J.D. Parent, D.C. Gami and O.T. Bloomer: "Thermodynamics and Transport Properties of Gases, Liquids and Solids", ASME, 180, McGraw-Hill (1959)

E-13) Eubanks, L.S. : Ph. D. Thesis Rice Inst. Houston, Texas (May 1957)

F-12) Fredensiund, A, J. Mallerup and O. Perssan: J. Chem. Eng. Data, 17, 4, 440 (1972)

F-13) Fredenslund, A. G. and A. Sather: J. Chem. Eng. Data, 15, 17 (1970)

F-17) Fisher, G.D. and T.W. Leland, Jr.: I. and E. C., Fundamenta's, 9 (4), 537 (1970)

F-18) Fredenslund, A. and G.A. Sather : I. and E. C., Fundamentals, \&, 718 (1969)

G-13) Grayson, H. G. and C.W. Streed: 第 6 回世界石油会境 更要論文集, 下巻, 273, 石油評論社 (1964)

G-14) Gunn, R.D. : A. I. Ch. E. J., 18 (1), 183 (1972)

H-16) 平田光䅹, 得田精二郎：化学工学, 31 (8), 759 (1967)

H-21) Hadden, S. T. : Chem. Eng. Progr., Symp. Ser., 49(7), 53 (1953)

H-22) Hadden, S. T. and H. G. Grayson: Hydrocarbon Process Petro: Refiner, 40, 207 (1961)

H-23) Hala, E., J. Pick, V. Fried and O. Vilim: "VaporLiquid Equilitrium", 2nd ed, Pergamon Press, Oxford (1967)

H-24) 平田光稳, 中原伯子: 化学工学, 28 (1), 2 (1964)

H-25) 平田光移, 須田精二郎 : 化学工学, 28 (5), 397 (1964)

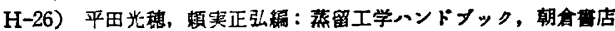
(1966)

H-27) 平田光稞, 白田利勝: 石油学会談, 10 (4), 41 (1967)

H-28) Hirata, M., S. Suda : Bull. Japan Petrol. Inst., 10, 20 (1968)

H-29) Hirschfelder, J. O., R. J. Buehler, H. A. McGee and J. R. Sutton: Ind. Eng. Chem., 50 (3), 375 (1958)

H-30) Hoffman, D.S., J.R. Welker, V.N.P. Rao and J.H. Weber: A.I.Ch.E.J., 10, 901 (1964)

H-31) Hoffman, D.S., J.R. Welker, R.E. Felt and J.H. Weber: A. I. Ch. E. J., 8, 508 (1962)

H-33) Hurt, L. J.: Ph. D. Thesis Rice Univ., Houston, Texas (1962)

H-34) 平田光理5：石油誌, 10 (4) 281 (1967)；11，(3) 195, (7) 555 (1968); 12 (9), 711, (12) 796, (12) 960 (1969); 13, (1) $45,(2), 127(1970)$

H-35) 平田光㮩, 中原伯子: 石油䒬, 9 (11), 879 (1966)

$\mathrm{H}-36$ ) 壮田光楆, 中原伯子: 石油夰, $12(1), 33$ (1969)

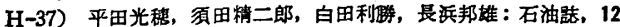
(10), 773 (1969)

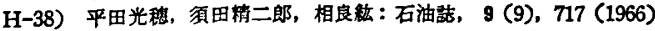

$\mathrm{H}-39$ ) 平田光稔, 白田利勝, 小野田忠雄: 石油誌, 10 (7), 440 (1967)

H-40) Mitsuho Hirata, Seijiro Suda : Bulletin of The Japan Petroleum Institute, Vol. 10, 20 (1968)

J-2) Jung, J., O. Knacke and D. Neuschatz: Chem.-Ing.-
Tech., 43, 112 (1971)

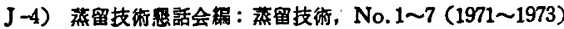

K-5) Kao, J. T. F.: J. Chem. Eng. Data, 15, 362 (1970)

K-6) Kay, W. B. : Ind. Eng. Chem., 30, 459 (1938)

K-10) Kay, W. B. and G. M. Rambosek: Ind. Eng. Chem., 45, 221 (1953)

K-15) Kay, W. B.: J. Chem. Eng. Data, 15, 46 (1970)

K-16) Kay, W. B. : J. Chem. Eng. Data, 16 (2), 137 (1971)

K-20) Kidnay, A.J, R.C. Miller and M.J. Hiza: $I$. and E. C. Fundamentals, 10 (3), 459 (1971)

K-25) Kohn, J.P. and W.F. Bradish : Paper presented at A. I. Ch. E. Meeting, Baltimore, Sept. (1957)

K-29) Konoki, K, K. Takeuchi, G. Kaminishi and T. Toriumi : J. Chem. Eng. Japan, 5 (2), 103 (1971)

K-36）上西玄一，鳥海通郎：工化，69 (2)，175 (1966)

K-37）片山俊，加藤目弘：化学工学, 35 (11), 1176 (1971)

K-38) Kaufmann, T.G.: I. and E. C., Fundamentals, 7, 115 (1967)

K-39) "Kellogg Equilibrium Charts" : The M.W. Kellogg Co, New York, Chem. Eng. Progr, 46, 20 (1950)

K-40) Kogan, W.B. and W.M. Fridman: Handbuch der Dampf-Flosigkeits-Gleichgewichte, VEB Deutscher Verlag der Wissen Shaften, Berlin (1961)

K-41）小島和夫, 加藤昌弘: 化学工学, 32 (9), 845 (1968)

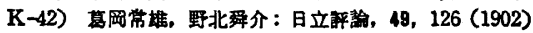

K-43) Kratochvil, J. J. Sobr, J. Matouš and J. Pick: Collection Czech. "Chem. Commun.”, 35, 3761 (1970)

L-15) Leach, J.W. P.S. Chappelear and T.W. Leland: A. I. Ch. E.J., 14, 568 (1968)

L-16) Lee, T. W., S. B. Wyatt, S. H. Desai and K.C. Chao: Preprints for Cryo. Eng. Conference B-4, Aug. 19, Cleveland Ohio, Case Western Univ. (1968)

L-18) Lee, B.I. and W.C. Edmister: A. I. Ch. E. J, 17 (6), 1412 (1971)

L-19) Leland, T. W.Jr., P.S. Chappelear and B. W. Gamson: A. I. Ch. E. J., 8, 482 (1962)

L-20) Leland, T. W. Jr. and P.S. Chappelear: Ind. Eng. Chem., 60, 15 (1968)

L-21) Lenoir, J. M. and G. A. White: Petro!. Ref, 37, 173 (1958)

L-22) Lenoir, J. M., B.C. Cajander and H. G. Hipkin: J. Chem. Eng. Data, 5, 251 (1960)

L-23) Lin, M.S. and. L. M. Naphthali : A. I. Ch. E. J., 9, 580 (1963)

M-5) Mathews, J.F. and D.C.D. Morey : Can. J. Chem. Eng, 49, 282 (1971)

M-19) Miller, R.C. A.J. Kidney and M. J. Hiza: $A$. I. Ch. E. J, 19 (1), 145 (1973)

M-21) Motard, R. L. and E.I. Organick: A. I.Ch. E. J., 6 , 39 (1960)

M-22) Muirbrook, N.K. and J.M. Prausnitz: A. I. Ch. E. J., 11 (6), 1092 (1965)

M-24）增田弘昭, 頼実正弘 : 化学工学, 32,820 (1968)

M-25) Mikolaj, P.G. and D. Lachhman: A. I. Ch. E. J., 17(2), 343 (1971)

N-1) Nagahama, K. and M. Hirata: J. Chem. Eng. Japan, ( (3), 205 (1971)

N-2) Nohka, J., E. Sarashina, Y. Arai and S. Saito: $J$. Chem. Eng. Japan, 6 (1), 10 (1973)

N-9）中原伯子, 平田光㮴：石油誌, 12, 33 (1969)

N-10) Nat. Gas Processois Assoc.: "Equilibrium Ratio Data Book", Trlsa, Okla. (1957)

$\mathrm{N}-11$ ） 日本材料学会高任力部門委員会：“高圧実影技街とその応用”， 丸嗛 (1969)

N-12）長浜邦婎, 須田精二郎, 白田利勝, 平田光稗：石油誌，14(4), 252 (1971)

0-6) Orye, R.S.: I. and E. C., Process Design and Deve$\operatorname{lop}_{7} 8,579$ (1969)

P-2) Pavlov, S. Y., V.A. Gorshkov, T. G. Zaikina, A. N. Bushn and V.V. Skorikova: Khim. Prom, (11), 810 (1970) 
P-3) Perillowx, C. J. and H. A. Deans: Ind, Eng, Chem, Fundamentals, 11 (1), 138 (1972)

P-13) Prodany, N.W. and B. Williams: J. Chem. Eng. Data, 16 (1), 1 (1971)

P-14) Prausnitz, J.M. C.A. Eckert, R. V. Orye and J.P. O'Conell: "Computer Calculation for Multicomponent Vapor-Liquid Equilibria", Erglewood Cleffs, N.J. Prentice-Hall (1967)

P-15) Prausnitz, J.M. and P.L. Chueh: "Computer CalcuJations for High Pressure Vapor-Liquid Equilibria", Prentice-Hall (1968)

P-16) Prausnitz, J. M.: "Molecular Thermod ynamics of Fluid Phase Equilibria", Prentice-Hall (1969)

P-17) Price, A.R., T. W. Leland and R. Kobayashi : Chem. Eng. Progr. Symp. Ser., 55, 13 (1959)

R-17) Reynes, J.A., M.J. Carles and J. Aubert: J. Chem. Phys, 67, 676-679 (1970)

R-18) Reynes, J.A. M. J. Carles and J. Aubert: J. Chem. Phys, 67, 680-683 (1970)

R-19) Reynes, J. A, M. J. Carles and J. Aubert: J. Chem. Phys; 67, 1526-1529 (1970)

R-20) Reynes, J.A., M. J. Carles and J. Aubert: J. Chem. Phys, 67, 1530-1533 (1970)

R-21) Reynes, J. A, M. J. Carles and J. Aubert: J. Chem. Phys., 67, 1534-1537 (1970)

R-22) Rhodes, H. L., W.E. Devaney and P.C. Tully: J. Chem. Eng. Data, 16 (1), 19-23 (1971)

R-23) Ribeirs, J. V. A. A. Susu and J.P. Kohn: J. Chem. Eng. Data, 17 (3), 279-283 (1972)

R-30) Rogers, B. L. and J. M. Prausnitz: J. Chem. Thermodynamics, 3, 211-216 (1971)

R-36) Redlich, O. and J.N.S. Kwong: Chem. Rev., 44, 233 (1949)

R-38) Robinson, D.B. and A.C. Saxena: Can. J. Chem. Eng. 47 (2), 69 (1969)

R-39) Ruhemann, M. and A. Harmens: Chem. Eng. Nov. 254 (1967)

R-40) Rowlinson, T.S. and I.D. Watson: Chem. Eng. Sci., 24, 1565, 1575 (1969)

S-1) Sagara, H., Y. Arai and S. Saito: J. Chem. Eng. Japan, 5 (4), 339 (1972)

S-3) Sage, B.H. W. N. Lacey and J.G. Schoafsma: Ind. Eng. Chem., 26, 103, 214 (1934)

S-25) Steckel, F. A. and N. Zinn: Zh. khim. Prom., 16 (8), 24 (1939)

S-28) Stein, F.P. and P.C. Proust: J. Chem. Eng. Data, 16 (4), 389 (1971)

S-32) Streett, W. B.: Cryogenics (Feb.), 27 (1965)

S-34) Streett, W.B. R.E. Sonntag and G. J\& Wylen: J. Chem. Phys, 40, 1390 (1964)

S-37) Streett, W. B. and J. L. E. Hill : J. Chem. Phys, 52, 1402 (1970)

S-40) Stutzman, L.F. and G.F. Brown: Chem. Eng. Progr, 45, 139 (1949)

S-42) Saito, S., G. Kaminishi, Y. Arai and S. Maeda: $J$. Chem. Eng. Japan, 1 (2), 109 (1968)

S-43）究藤正三郎：蒸留技術, 2 (1), (2), (3) (1972), 2 (4) (1973)

S-44) Saito, S., H. Sagara and Y. Arai : J. Chem. Eng. Japan, 5 (4), 339 (1972)

S-45) Sarashina, E, Y. Arai and S. Saito: J. Chem. Eng. Japan, 6 (2), 120 (1973)

S-46) Scott, R. B.: “Cryogenic Engineering”, D. Van Nostrand Co., Inc. Princeton, New Jersey

S-47) Shelton, R. J. and R.M. Wood: Chem. Engineer, 46, CE 68:(1965)

S-48) Shelton, R. J.: Chem. Engineer, 46, CE 385 (1968)

S-49) Simmon, R. and J.E. Briggs: A. I: Ch. E. J., 10, 548 (1964)

S-50) Singh, S.P. and P.K. Mukhopadhyay: A. I. Ch. E. J.
18 (6), 1171 (1972)

S-51) Soave, C.: Chem. Eng. Sci, 27, 1197 (1972)

S-52) Sood, S.K. and G. G. Haselden: Cryogenics, 10, 199 (1970)

S-53) Spear, R.R., R. L. Robinson, Jr. and C.C. Kwong : I. and E. C., Fundamentals, 10 (4), 588 (1971)

S-54) Stotler, H.H. and M. Benedict: Chem. Eng. Symp. ser. 49, (6) 25 (1953)

S-55) Streett, W. B. and C.H. Jones: J. Chem. Phys, 42 (11), 3989 (1965)

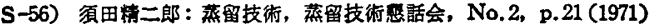

S-58) Streett, W.B.: Private Communication, August (1971)

T-3) Thorogood, R.M. and G.G. Haselden: Brit. Chem. Eng., 8 (9), 623 (1963)

T-10) Trust, D.B. and F. Kurata: A. I. Ch. E. J, 17 (2), 415 (1971)

T-11) Trust, D.B. and F. Kurata : A. I. Ch. E. J. 17 (1), 86 (1971)

T-12) Trinh, B.T., R.S. Ramalho and S. Kaliaguine: Can. J. Chem. Eng., 50. (6), 771 (1972)

T-17) Toyama, A., P.S. Chappelear, T. W. Leland and R Kobayashi: Adv, in Cryogenic Engn 7, 106 (1961)

V-1) Vairogs, J, A.J. Klekers and W.C. Edmister: $A . I$. Ch. E. J., 17 (2), 308 (1971)

V-7) Van Horn, L. D. and R. Kobayashi : A. I. Ch. E. J. 14, 92 (1968)

V-8) Vera, J.H. and J.M. Prausnitz: Chem. Eng. Sci., 26, 1772 (1971)

W-8). Wichterle, I. and R. Kobayashi: J. Chem. Eng. Data, 17 (1), 9 (1972)

W-16) Wenzel, H. and S. Peter: Chem.-Ing.-Tech., 43 (15), 856 (1971)

W-17) White, G.Y.: "Experimental Techniques in Low Temperature Physics", Oxford University Press(1968)

W-18) Wichterle, I., Z.W. Salsturg, P.S. Chappelear and R. Kobayashi: Chem. Eng. Sci., 26, 1141 (1971)

W-19) Wilson, G. M. : Adv. Cryogenic Eng., 9, 168 (1964)

W-20) Winn, F. W.: Chem. Eng. Progr. Symp. Ser. 48, 121 (1952)

Y-7) 頼実正弘, 吉村尚真, 仵岡弘睠：化学工学, 34 (9), 953 (1970)

Y-8) Yudovich, A, R. L. Robinson and K. C. Chao: A. L. Ch. E. J, 17, 5, 1152 (1971)

Y-12）頼実正弘，吉村尚真：ヶミカルェンジニフリング, 13 (12), (1968), $14(1-6),(1969)$

Y-13）頼实正弘，件岡弘勝：石油学会誌，12，(1) 14 (1969)

Y-14）頼実正弘：化学機做技術，17，1 (1965)

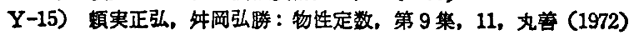

Y-16) Yorizane, M. S. Yoshimura, H. Masuoka and M. Nakamura: J. Chem. Eng. Japan, 4 (1), 10 (1971)

Y-17) Yorizane, M, S. Yoshimura and N. Shiki: Memories of the Faculty of Eng., Hiroshima Univ, 3 (3), 9 (1969)

Y-18）頼英正弘, 吉村尚真：ケミカルエンシニャリンク, 16 (1), (1971)

Y-19）頼実正弘，吉村尚真，舛岡弘勝，江藤祐一，中子敬夫，船田 一郎：化学工学協会第 4 回秋委大会前刷集, 157 (1970)

Y-20) 頼実正弘, 吉村尚真, 舛岡弘勝: 化学工学, 30 (12), 1093 (1966)

Y-21）頼実正弘，吉村尚凘，外岡弘膦，中隆之：化学工学， $35(6)$, 691 (1971)

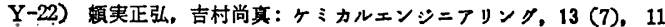
(1968), 16 (1), 10 (1971)

Y-24) Yorizane, M., S. Yoshimura and H. Masuoka: Bull. Japan Petrol. Inst, 14 (1), 105 (1972)

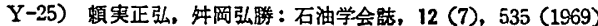

Z-1) Zeininger, H.: Chem. Ing. Tech, 44 (9), 607 (1972)

Z-4) Zellner, M.G, and J.M. Prausnitz: I. and E. C., Fundamentals, 9 (4), 549 (1970)

Z-3) Zenner, G.H. and L.I. Dana: Chem. Eng. Progr. Symp. Ser. (Thermodynamics), 59, 36 (1963) 